\title{
In Situ Compatibilized Polymer Blends of Phenoxy and ABS
}

\author{
SUE-HUA CHEN and FENG-CHIH CHANG * \\ Institute of Applied Chemistry, National Chiao-Tung University, Hsinchu, Taiwan, Republic of China
}

\begin{abstract}
SYNOPSIS
The combination of styrene-acrylonitrile-glycidyl methacrylate (SAG) reactive copolymer and sodium lauryl sulfonate catalyst is able to function as an effective in situ compatibilizer for the otherwise immiscible and incompatible polymer blends of phenoxy and acrylonitrilebutadiene-styrene (ABS). The copolymer formed from the reaction between phenoxy and SAG under melt blending conditions tends to reduce interfacial tension in the melt and results in finer morphological domains of the blends. The presence of this in situ formed compatibilizer also raises the interphase adhesion of the blends and results in significant improvements in mechanical properties. (c) 1994 John Wiley \& Sons, Inc.
\end{abstract}

\section{INTRODUCTION}

Polyhydroxy ether of bisphenol A is a high molecular weight thermoplastic made from bisphenol $\mathrm{A}$ and epichlorohydrin that is tough, ductile, and possesses high cohesive strength and good impact resistance. Polymer blends with phenoxy as a component have been studied previously including in combination with polycarbonate, ${ }^{1-5}$ various polyesters, ${ }^{6-13}$ polymethylmethacrylate, ${ }^{14-16}$ and others. ${ }^{17-22}$ The interchange reactions between phenoxy and polycarbonate or polyesters are well-recognized and the resulted interchange reaction products improve miscibility of these blends or even result in miscible blends. ${ }^{1,3,6,7,9-12,18}$ Acrylonitrile-butadiene-styrene copolymer (ABS) is a tough thermoplastic with balanced properties and its blends with numerous polymers have been extensively investigated previously. However, due to poor compatibility between phenoxy and styrenic polymers, very little or none of the literature have reported on polymer blends between phenoxy and styrenic polymers such as polystyrene or ABS.

Methods to improve blend compatibility between two immiscible and incompatible polymers have been a subject of considerable research activities that have been summarized recently by Xanthos ${ }^{23}$ and

* To whom correspondence should be addressed. Journal of Applied Polymer Science, Vol. 51, 955-965 (1994) (C) 1994 John Wiley \& Sons, Inc.
Utracki. ${ }^{24}$ It is well known that block or graft copolymers capable of specific interactions with the blend constituents can function as blend compatibilizers. These copolymers may be considered as true interfacial agents because they tend to concentrate at the interface and act as an emulsifier. Alternatively, a copolymer $\mathrm{A}-\mathrm{X}$ ( $\mathrm{X}$ represents reactive groups) will react with blend component $B$ to form A-X-B copolymers under melt conditions that will function as a nonspecific type compatibilizer for a polymer pair of $\mathrm{A}$ and $\mathrm{B}$. Before reaction, copolymer A-X itself cannot be considered as a compatibilizer for a polymer blend of A and B.

Most previously reported literature in the area of in situ compatibilization has been mainly concentrated on maleic anhydride-containing copolymers. Copolymers with glycidyl methacrylate (GMA) monomer are able to react with polymers containing phenolic- $\mathrm{OH},{ }^{25}-\mathrm{COOH},{ }^{26,27}$ or amine ${ }^{28,29}$ endgroups under melt blending conditions and function as in situ compatibilizers of the blends. Catalyst may or may not be required depending on the reactivity and the extent of the desired reaction. A series of continuous investigations on in situ compatibilization of various polymer blends using the GMA-containing copolymers was carried out in this lab. This article reports the polymer blends of phenoxy and ABS by employing styrene-acrylonitrile-glycidyl methacrylate (SAG) as the in situ compatibilizer. The major difference between this and our previous published literature is that the reactive functional groups of the blend component, aliphatic-OH, are 
distributed along the main chain and they are relatively less reactive.

\section{EXPERIMENTAL}

\section{Materials}

UCAR Phenoxy resin, PKHH, was obtained from Union Carbide Corporation. ABS with $18 \%$ rubber content, D-120 grade, was obtained from Grand Chemical Company of Taiwan. SAG with monomer ratio, Sty/AN/GMA $=65 / 25 / 5$, was prepared by suspension polymerization and the detailed procedures were described previously. ${ }^{25}$ Sodium lauryl sulfonate used as a melt reaction catalyst between SAG and phenoxy resin was obtained from Merck.

\section{Melt Blending and Specimen Preparation}

The blends were prepared using a $20-\mathrm{mm}$ Welding Engineering twin-screw extruder with L/D of 48 and counter-rotating intermeshing screws. The blended pellets were molded into $0.32-\mathrm{cm}$ test specimens on an Arburg 3-oz injection-molding machine.

\section{Torque Versus Time Measurements}

To verify the reaction between SAG and phenoxy resin based on the viscosity increase, $30 \mathrm{~g}$ of sample were tested at $230^{\circ} \mathrm{C}$ and $60 \mathrm{rpm}$ in a BrabenderPlastic-Corder.

\section{Characterizations}

Standard Izod impact (ASTM-D256), tensile (ASTM-D638), instrumental falling weight impact, and critical strain energy release rate $\left(G_{c}\right)$ tests were carried at ambient conditions as described previously. ${ }^{30-32}$ Morphologies of the impact fracture surfaces were investigated by scanning electron microscopy (SEM) using a Mode S-570 from Hitachi Co. of Japan. Melt index (MI) of the blends were measured at $230^{\circ} \mathrm{C}$ and $3.8 \mathrm{~kg}$ load according to AS'TM-D1238.

\section{RESULTS AND DISCUSSION}

\section{Fundamental of In Situ Compatibilization}

A specific type compatibilizer is a synthesized block or graft nonreactive copolymer with segments iden- tical or miscible with the blend components and its structure and quantity are unchanged and independent of blending conditions. On the contrary, an in situ reactive compatibilizer is a copolymer containing reactive functional groups that is able to react with one (or both) of the blend component to form a copolymer and act as a compatibilizer. The content and structure of an in situ compatibilizer vary with the content of the reactive groups, temperature, time, mixing efficiency, and presence of catalyst. Therefore, this type of compatibilizer is considered a nonspecific type compatibilizer. Before reaction, the SAG copolymer itself cannot act as a phase compatibilizer for the polymer blends of ABS and phenoxy resin. However, the reaction products between the epoxide groups and the hydroxyl groups of phenoxy resin will form various SAG-phenoxy copolymers that will function as the nonspecific compatibilizer. An excessive reaction between SAG and phenoxy resin may result in cross-linked copolymer and become too bulky and too high in viscosity to be effective. Therefore, the optimized content of the in situ formed copolymer is essential to achieve the greatest performance of the resulted blend products. This can be accomplished through proper adjustment of the GMA content in the SAG copolymer, the presence of a suitable catalyst, and proper control of the blending conditions.

The epoxide groups in SAG can react with hydroxyl or carboxylic acid endgroups of polyesters ${ }^{25-27}$ and amine endgroups of nylons. ${ }^{29}$ The reactivity between epoxide and phenolic- $\mathrm{OH}$ is considerably greater than aliphatic- $\mathrm{OH}$ due to the acidity difference. Therefore the reaction between the epoxide groups in SAG and aliphatic-OH in the phenoxy resin is relatively slower even though significantly greater numbers of the reactive aliphatic- $\mathrm{OH}$ groups are present. The ethyl triphenylphosphonium bromide catalyst was found to be an effective catalyst in promoting reaction between phenolic- $\mathrm{OH}$ and carboxylic acid ${ }^{25-27}$ but was found less effective in the current system. A new catalyst, sodium lauryl sulfonate, has been discovered to be effective to promote reaction between epoxide and the less reactive aliphatic- $\mathrm{OH}$ in melt conditions and was selected in this study.

The lightly reacted copolymer is the one that can act as an effective phase compatibilizer. Because both SAG and phenoxy resin possess multiple reactive groups, to a greater extent the reaction tends to form a cross-linking network and becomes less effective as a phase compatibilizer. The reaction between SAG copolymer and phenoxy resin can be 
expressed by the following equation:<smiles>CCC(O)COc1ccc(C(C)(C)c2ccc(OC(C)(C)C)cc2)cc1</smiles>

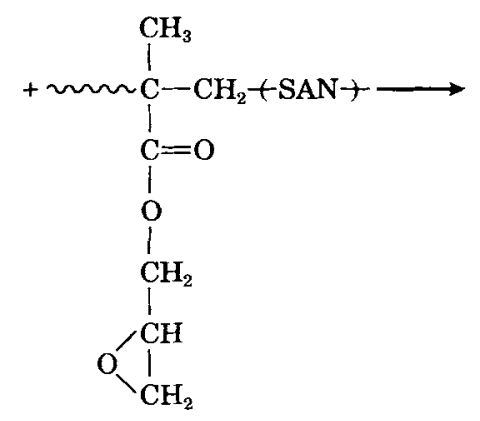

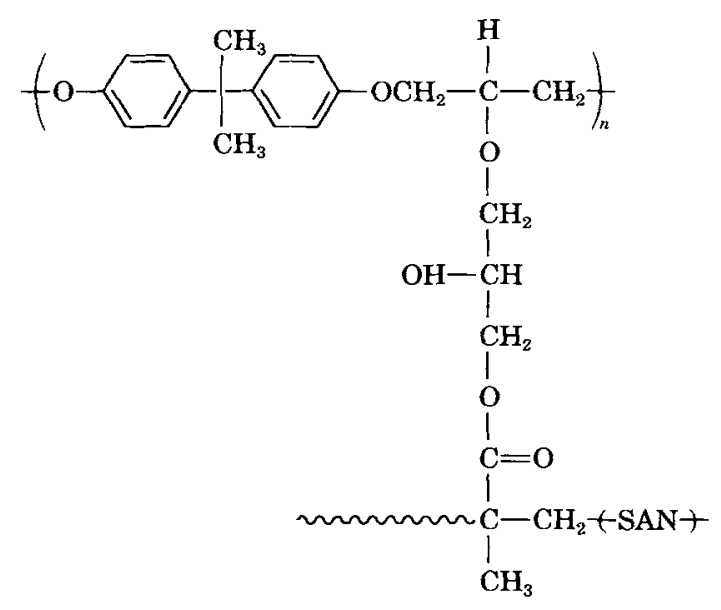

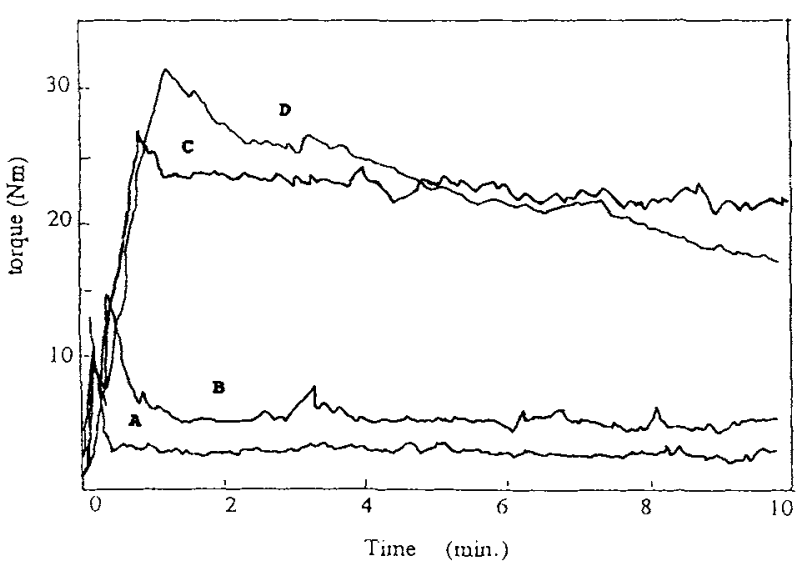

Figure 1 Torque vs. time at $230^{\circ} \mathrm{C}$ and $60 \mathrm{rpm}$ : (A) Phen $/ \mathrm{SAG}=50 / 50$; $(\mathrm{B})$ Phen $/ \mathrm{SAG} / \mathrm{Cat}=50 / 50 / 0.1$; (C) Phen/ABS $/ \mathrm{SAG}=75 / 25 / 5$; (D) Phen $/ \mathrm{ABS} / \mathrm{SAG}$ / Cat $=75 / 25 / 5 / 0.05$.
Table I Melt Indexes of Various Phen/ABS Blends

\begin{tabular}{lr}
\multicolumn{1}{c}{ Composition } & MI $(\mathrm{g} / 10 \mathrm{~min})$ \\
\hline & \\
Phenoxy & 18.9 \\
ABS & 4.2 \\
SAG & 30.3 \\
Phen/ABS $=75 / 25$ & 13.4 \\
Phen/ABS/SAG $=75 / 25 / 5$ & 9.9 \\
Phen/ABS/SAG/Cat $=75 / 25 / 5 / 0.02$ & 8.1 \\
Phen/ABS/SAG/Cat $=75 / 25 / 5 / 0.05$ & 6.2 \\
Phen/ABS/SAG/Cat $=75 / 25 / 5 / 0.10$ & 5.9 \\
Phen/ABS/SAG $=75 / 25 / 2$ & 10.4 \\
Phen/ABS/SAG/Cat $=75 / 25 / 2 / 0.05$ & 8.0 \\
Phen/ABS/SAG $=75 / 25 / 10$ & 4.9 \\
Phen/ABS/SAG/Cat $=75 / 25 / 10 / 0.05$ & 4.1 \\
Phen/ABS $=50 / 50$ & 9.0 \\
Phen/ABS/SAG $=50 / 50 / 5$ & 7.1 \\
Phen/ABS/SAG/Cat $=50 / 50 / 5 / 0.02$ & 6.6 \\
Phen/ABS/SAG/Cat $=50 / 50 / 5 / 0.05$ & 4.8 \\
Phen/ABS/SAG/Cat $=50 / 50 / 5 / 0.10$ & 4.2 \\
Phen/ABS/SAG $=50 / 50 / 2$ & 8.6 \\
Phen/ABS/SAG/Cat $=50 / 50 / 2 / 0.05$ & 6.3 \\
Phen/ABS/SAG $=50 / 50 / 10$ & 2.8 \\
Phen/ABS/SAG/Cat $=50 / 50 / 10 / 0.05$ & 1.9 \\
\hline
\end{tabular}
load.

Cat, sodium lauryl sulfonate; conditions, $230^{\circ} \mathrm{C}$ and $3.8-\mathrm{kg}$

\section{Processability}

The incompatibility between ABS and phenoxy resin resulted in compounding difficulties such as die swelling and melt fracture during blending extrusion. The presence of SAG in the in situ com-

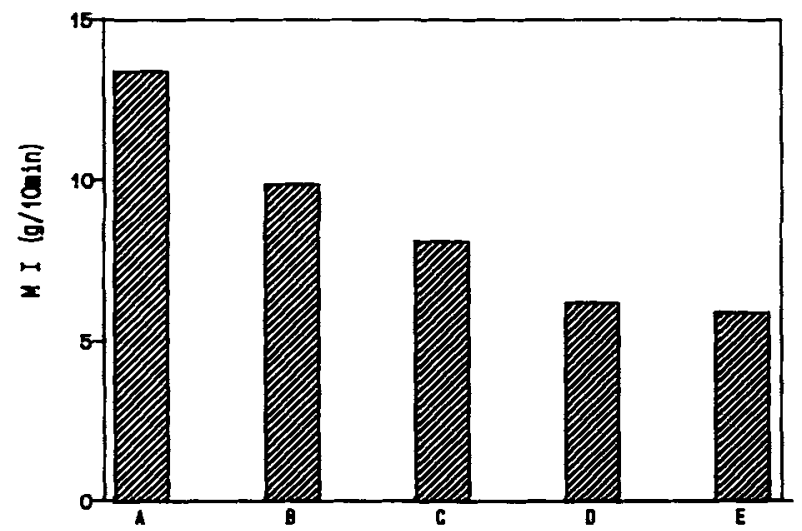

Figure 2 Melt indexes of various Phen/ABS blends: (A) Phen $/ \mathrm{ABS}=50 / 50$; (B) Phen $/ \mathrm{ABS} / \mathrm{SAG}=75 /$ $25 / 5$; (C) Phen $/$ ABS $/ \mathrm{SAG} /$ Cat $=75 / 25 / 5 / 0.02$; (D) Phen $/$ ABS $/$ SAG $/$ Cat $=75 / 25 / 5 / 0.05 ;(E)$ Phen/ABS/ $\mathrm{SAG} / \mathrm{Cat}=75 / 25 / 5 / 0.10$. 


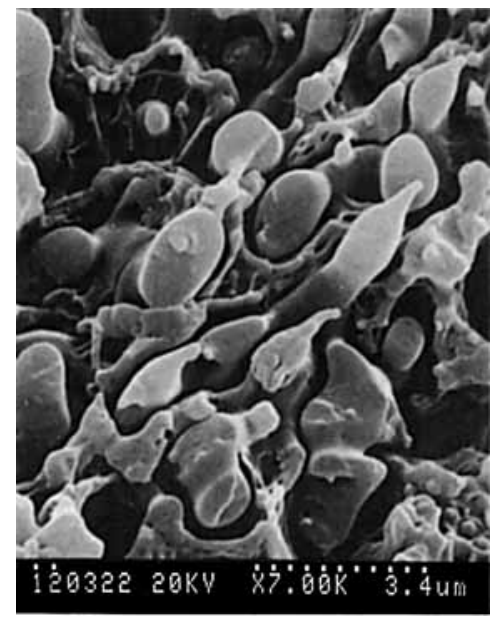

A. Phen $/ A B S=75 / 25$

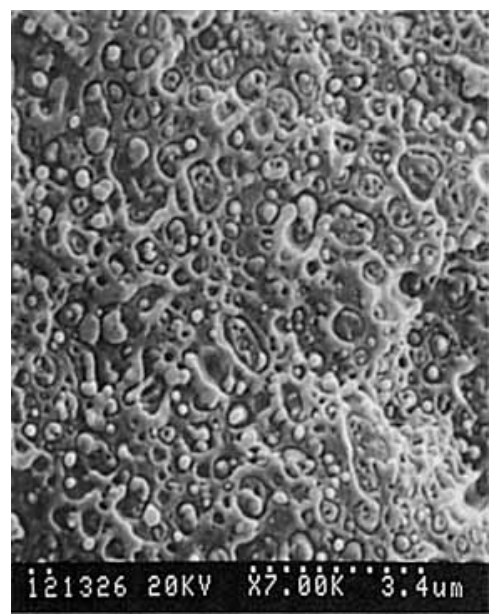

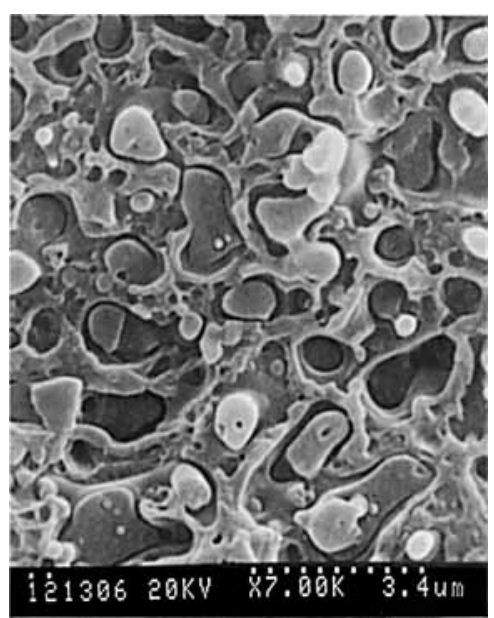

B. Phen $/$ ABS $/$ SAG $=75 / 25 / 5$

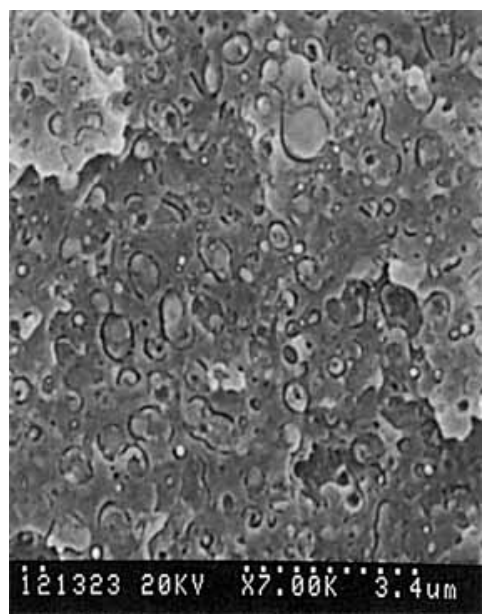

C. Phen $/ \mathrm{ABS} / \mathrm{SAG} / \mathrm{Cat}=75 / 25 / 5 / 0.02$

D. Phen $/$ ABS $/$ SAG $/$ Cat $=75 / 25 / 5 / 0.05$

Figure 3 SEM micrographs of the fracture surfaces of various Phen/ABS $75 / 25$ blends: (A) Phen $/ \mathrm{ABS}=75 / 25$; (B) Phen $/ \mathrm{ABS} / \mathrm{SAG}=75 / 25 / 5$; (C) Phen $/ \mathrm{ABS} / \mathrm{SAG} / \mathrm{Cat}$ $=75 / 25 / 5 / 0.02$; (D) Phen $/ \mathrm{ABS} / \mathrm{SAG} / \mathrm{Cat}=75 / 25 / 5 / 0.05$; (E) Phen $/ \mathrm{ABS} / \mathrm{SAG}=75 /$ 25/2; (F) Phen $/$ ABS $/$ SAG $/$ Cat $=75 / 25 / 2 / 0.05$; (G) Phen $/$ ABS $/$ SAG $=75 / 25 / 10$; (H) Phen $/$ ABS $/$ SAG $/$ Cat $=75 / 25 / 10 / 0.05$.

patibilizer reduced the above-mentioned processing difficulties. The presence of SAG and catalyst essentially solved most of these problems although a slightly higher torque was encountered as would be expected due to higher viscosity. Therefore, the in situ SAG compatibilizer with catalyst is able to convert a well-known incompatible $\mathrm{ABS} /$ phenoxy blend into a compatible one. 


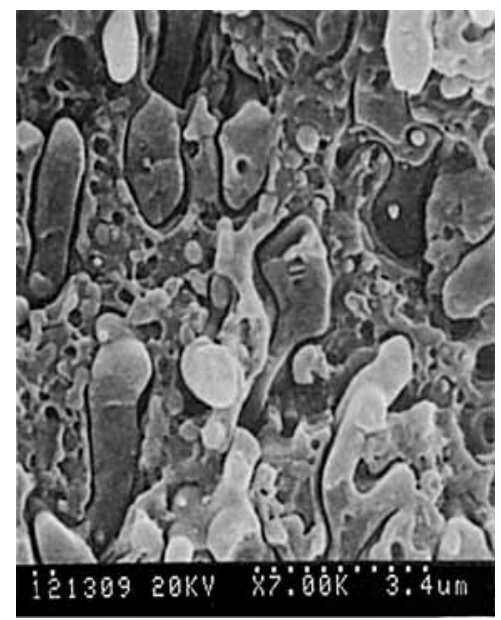

E. Phen $/$ ABS $/$ SAG $=75 / 25 / 2$

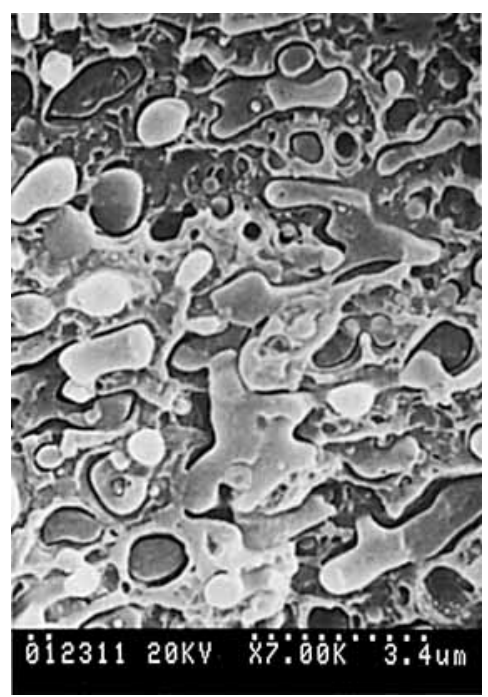

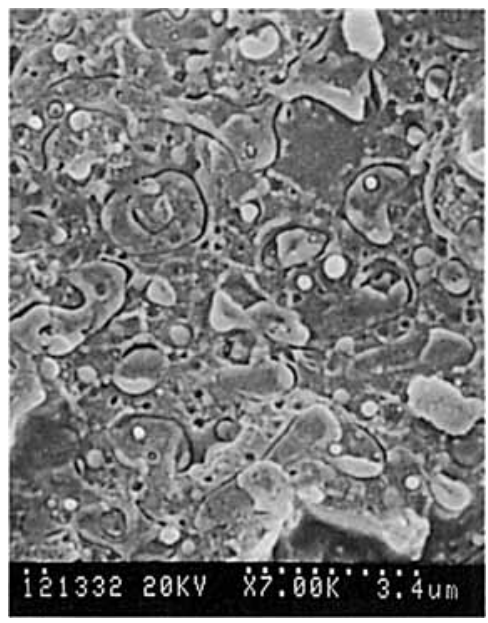

F. Phen $/$ ABS $/$ SAG $/$ Cat $=75 / 25 / 2 / 0.05$

Figure 3 (Continued from the previous page)

\section{Torque Versus Time}

Curve A of Figure 1 shows the torque of the mixture, Phen/SAG 50/50, where the torque remains fairly constant after $1 \mathrm{~min}$ with fairly low value (less than 3 Newton meters). Therefore the reaction between the epoxide groups from SAG and the aliphatic-OH from phenoxy resin is insignificant. The torque of this blend increases nearly eight times to $23 \mathrm{Nm}$ after one min and remains at this level steadily with the presence of $0.1 \mathrm{phr}$ of catalyst. These observed results imply that the catalyst employed is very effective to promote the reaction. The constant torque

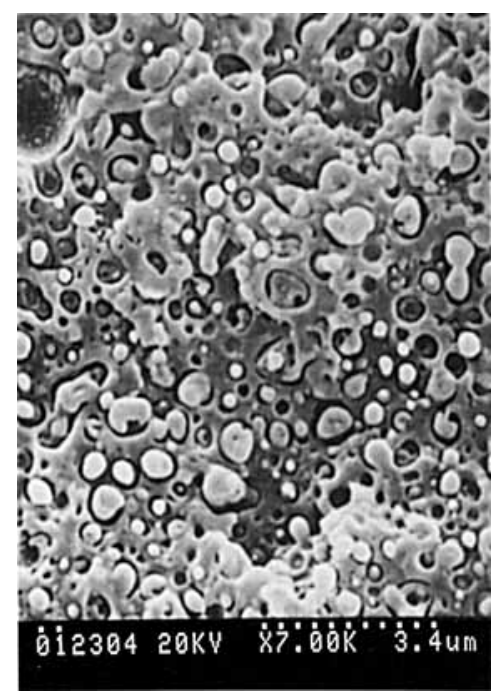

after the initial jump (Fig. 1, curve B) indicates that the catalyst may deactivate due to thermal decomposition, otherwise, a continuous increase of torque value should have been obtained. Curves $C$ and $D$ of Figure 1 again show significantly higher torque for the catalyst-containing blend than the corresponding blend containing no catalyst. 'The presence of the decomposed catalyst may cause ABS degradation and results in gradual torque decline after the initial jump as shown in curve D of Figure 1. The viscosity increase can be attributed undoubtedly to the molecular weight increase or even cross-linking if the degree of the anticipated reaction between 


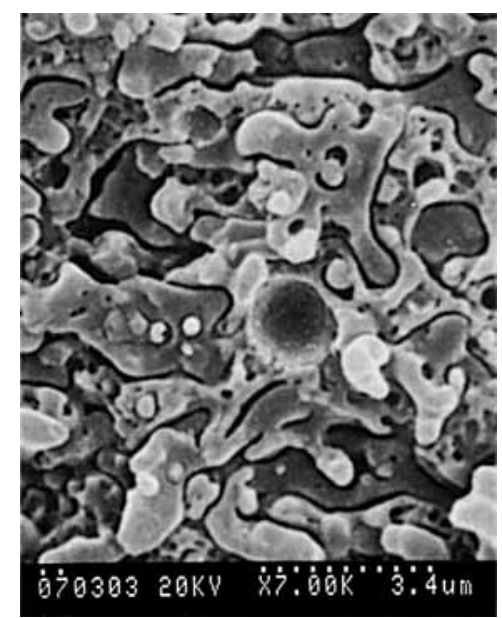

A. Phen $/ \mathrm{ABS}=50 / 50$

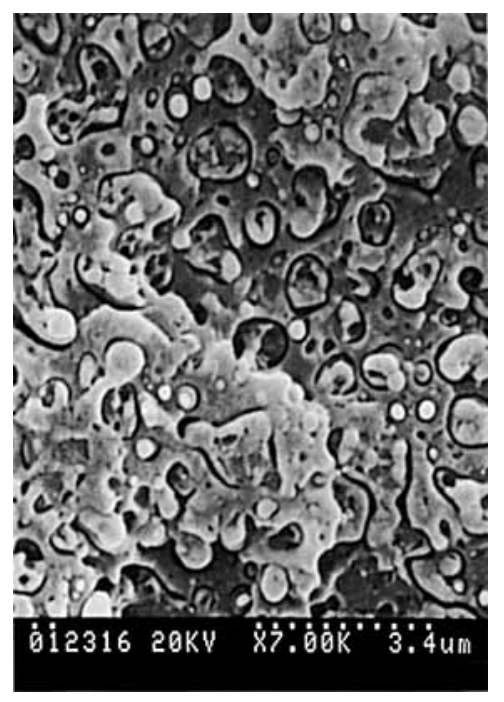

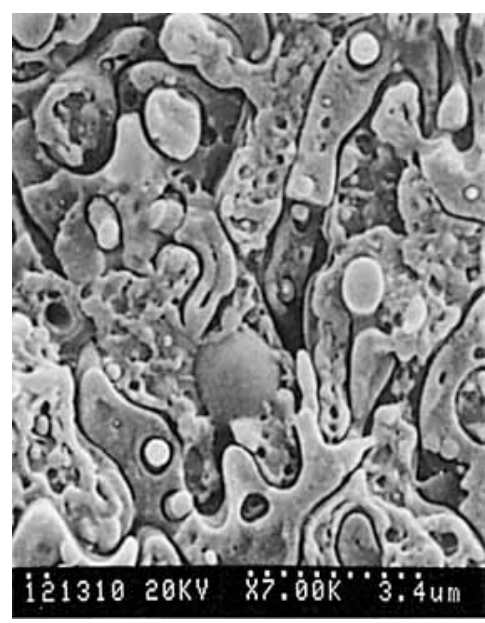

B. Phen $/ \mathrm{ABS} / \mathrm{SAG}=50 / 50 / 5$

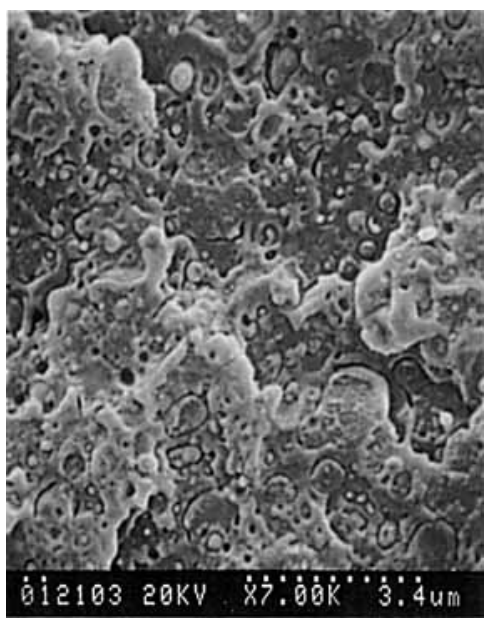
C. Phen $/$ ABS $/$ SAG $/$ Cat $=50 / 50 / 5 / 0.02$
D. Phen $/$ ABS $/$ SAG $/$ Cat $=50 / 50 / 5 / 0.05$

Figure 4 SEM micrographs of the fracture surfaces of various $P$ hen/ABS $50 / 50$ blends: (A) Phen $/ \mathrm{ABS}=50 / 50$; (B) Phen $/$ ABS $/ \mathrm{SAG}=50 / 50 / 5$; (C) Phen $/ \mathrm{ABS} / \mathrm{SAG} / \mathrm{Cat}$ $=50 / 50 / 5 / 0.02 ;(D)$ Phen $/$ ABS $/$ SAG $/$ Cat $=50 / 50 / 5 / 0.05$.

SAG and phenoxy is too high. Table I summarizes the melt indexes of the matrices, compatibilizer, and blends. Figure 2 illustrates the effect due to the presence of SAG and catalyst of various Phen/ABS
$75 / 25$ blends. The blend with $5 \mathrm{phr}$ SAG decreases MI from 13.4 to 9.9 and the presence of catalyst further reduces the MI of the blends to $8.1-5.9$ depending on the amount of catalyst. Similar results 


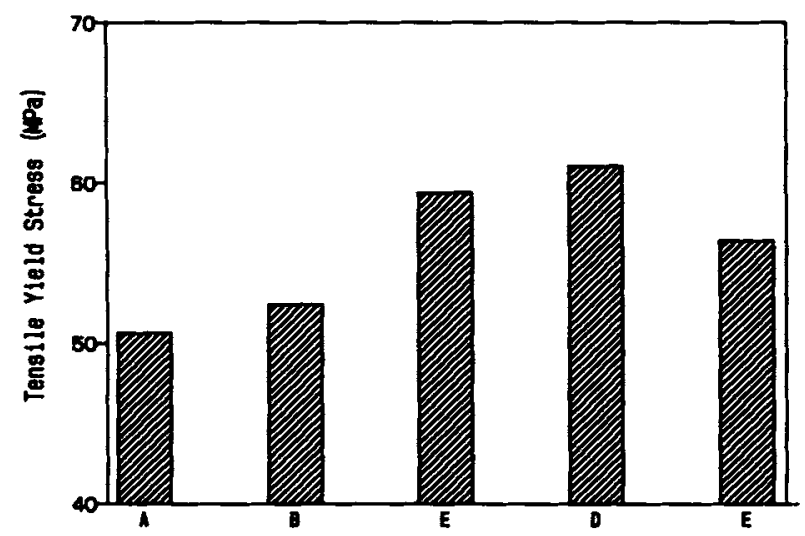

Figure 5 Tensile yield stress of various $P$ hen / ABS 75/ 25 blends: (A) Phen/ABS = 75/25; (B) Phen/ABS/SAG $=75 / 25 / 5$; (C) Phen $/$ ABS $/$ SAG $/$ Cat $=75 / 25 / 5 / 0.02$; (D) Phen /ABS $/$ SAG $/$ Cat $=75 / 25 / 5 / 0.05$; (E) Phen/ ABS $/ \mathrm{SAG} / \mathrm{Cat}=75 / 25 / 5 / 0.10$.

were also obtained from Phen/ABS 50/50 blends (Table I).

\section{SEM Morphologies}

Figure 3(A) shows the SEM micrograph of the fracture surface of the noncompatibilized Phen/ABS $75 / 25$ blend. This noncompatibilized blend shows relatively coarser domain of the dispersed ABS phase and the adhesion between ABS and phenoxy matrix is rather poor. The presence of $5 \mathrm{phr}$ of $\mathrm{SAG}$ in the blend only slightly reduces the domain size [Figure 3(B)]. The presence of SAG and catalyst significantly reduces the domain dimension of the

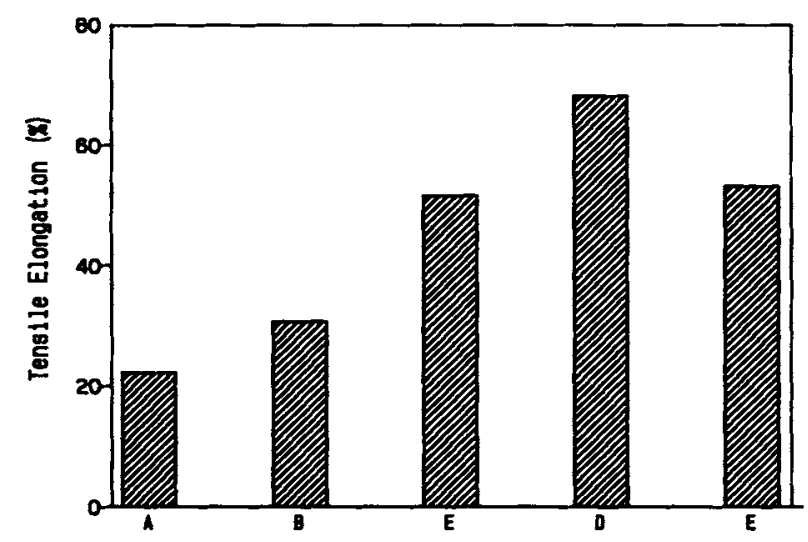

Figure 6 Tensile elongation to break of various Phen / ABS 75/25 blends: (A) Phen /ABS $=75 / 25$; (B) Phen/ ABS $/$ SAG $=75 / 25 / 5$; (C) Phen $/$ ABS $/$ SAG $/$ Cat $=75 /$ $25 / 5 / 0.02$; (D) Phen $/$ ABS $/$ SAG $/$ Cat $=75 / 25 / 5 / 0.05$; (E) Phen $/$ ABS $/$ SAG $/$ Cat $=75 / 25 / 5 / 0.10$.

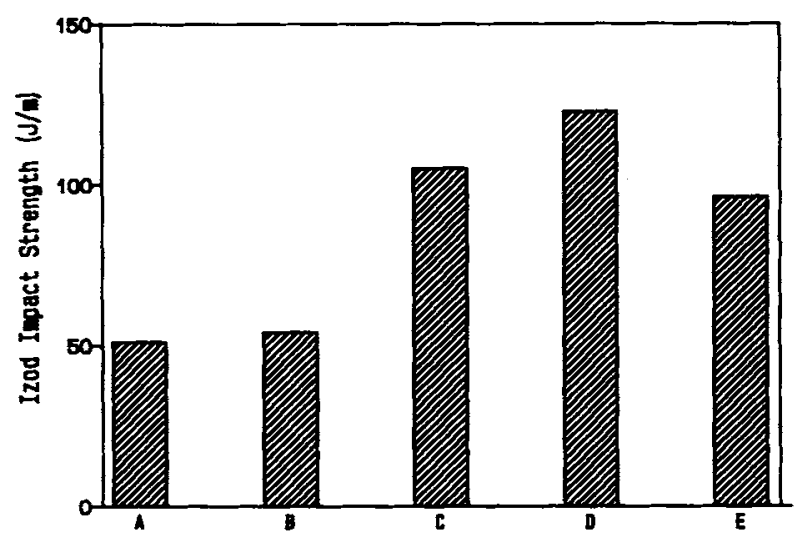

Figure 7 Izod impact strengths of various Phen/ABS 75/25 blends: (A) Phen $/ \mathrm{ABS}=75 / 25$; (B) Phen/ABS/ $\mathrm{SAG}=75 / 25 / 5$; (C) Phen $/ \mathrm{ABS} / \mathrm{SAG} / \mathrm{Cat}=75 / 25 /$ 5/0.02; (D) Phen $/$ ABS $/$ SAG $/$ Cat $=75 / 25 / 5 / 0.05$; (E) Phen $/$ ABS $/$ SAG $/$ Cat $=75 / 25 / 5 / 0.10$.

dispersed ABS phase [Fig. 3(C,D)]. This in situ formed copolymer has an SA segment and phenoxy segment and tends to reside at the interface to reduce melt interfacial tension and therefore functions as a compatibilizer between phenoxy and ABS. When the SAG content in the blend is increased to $10 \mathrm{phr}$, the domain size of the ABS phase does not show any significant change [compare Fig. $3(\mathrm{~B}, \mathrm{G})]$. With the presence of 0.05 phr of catalyst but varying $S A G$ content, the blend with 5 phr [Fig. 3(D)] has the finest morphology, then 10 phr [Fig. 3(H)] and 2 phr [Fig. 3(F)]. As mentioned earlier, too much reacted copolymer product is too bulky and less effective as a compatibilizer. Figure 4 shows the micrographs of various Phen/ABS 50/50 blends and a very similar trend with the same conclusion can be drawn.

\section{Tensile Properties}

Only the tensile yield stress and elongation to break of various Phen/ABS 75/25 blends will be presented here to demonstrate the effect of SAG and catalyst in these blends. Figure 5 clearly shows the yield stress increase due to the presence of SAG and catalyst. The blend with the greatest amount of catalyst employed in this study $(0.10 \mathrm{phr})$ does not follow the trend, it actually decreases significantly. This result is consistent with the previous domain size of the ABS dispersed phase. Figure 6 illustrates the tensile elongation (indication of tensile toughness) from the same set of blends and again a similar trend as in Figure 5 was obtained. 


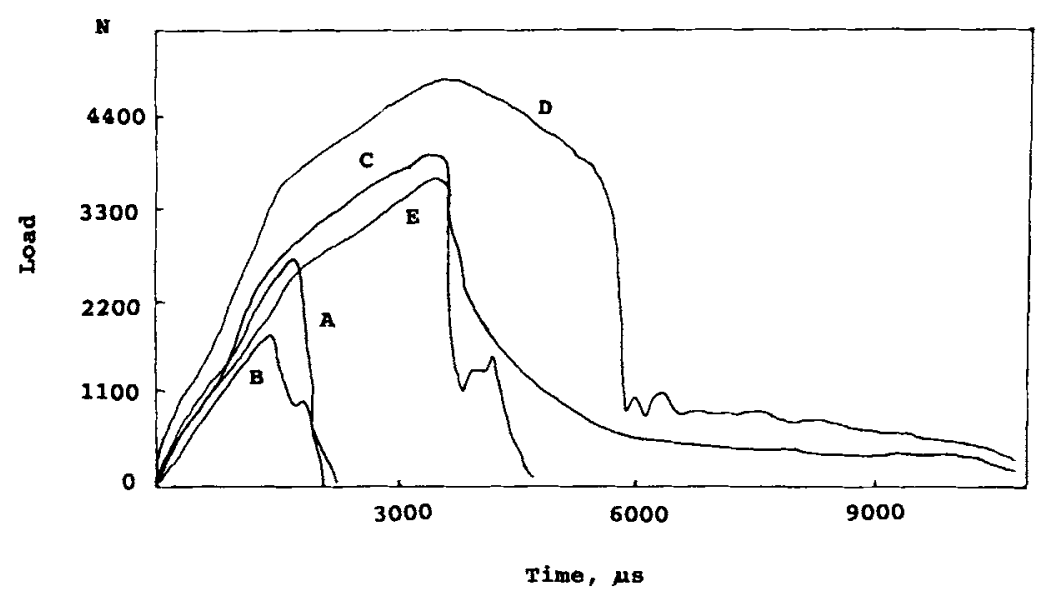

Figure 8 Instrumental falling weight impact load-time curves of various Phen / ABS $75 / 25$ blends: (A) Phen $/$ ABS $=75 / 25$; (B) Phen $/$ ABS $/$ SAG $=75 / 25 / 5$; (C) Phen/ ABS $/$ SAG $/$ Cat $=75 / 25 / 5 / 0.02 ;(D)$ Phen $/$ ABS $/$ SAG $/$ Cat $=75 / 25 / 5 / 0.05 ;($ E) Phen $/$ $\mathrm{ABS} / \mathrm{SAG} / \mathrm{Cat}=75 / 25 / 5 / 0.10$.

\section{Izod Impact Properties}

Figure 7 shows the Izod impact strength from the same set of blends. The blend with 5 phr SAG shows only slight improvement over the noncompatibilized blend (compare (A) and (B), Fig. 7). The increase of impact strength is very substantial when the blend contains both SAG and catalyst (C, D, and E of Fig. 7). Similar to the tensile properties, excessive catalyst content actually decreases its impact strength slightly.

\section{Instrumental Falling Weight Impact}

Figure 8 illustrates the instrumental falling weight impact load versus time curves from the same set of blends. Toughness order is essentially identical to Izod impact strength except the blends with and without containing SAG (curves A and B). The fracture energy of the noncompatibilized Phen/ABS $75 / 25$ blend is slightly higher than the one containing an additional $5 \mathrm{phr}$ of SAG. Figure 9 shows the total impact energies of those blends shown in Figure 8 . Figure 10 shows the photographs of the impacted specimens. Only two specimens from the blends containing SAG and catalyst are in ductile fracture mode. Figure 11 shows the instrumental falling weight load versus time curves from various Phen / ABS 50/50 blends. A similar trend as in Figure 9 was also obtained.

\section{Critical Strain Energy Release Rate, Gc}

The Gc derived from the basic fracture mechanics in terms of energy absorbed by the conventional Izod impact test has been previously developed. ${ }^{33,34}$ The energy absorbed by the impact at fracture rather than at the maximum load is assumed to deform in a total elastic manner so that the compliance, $C$, is a function of crack length and the geometry. Thus,

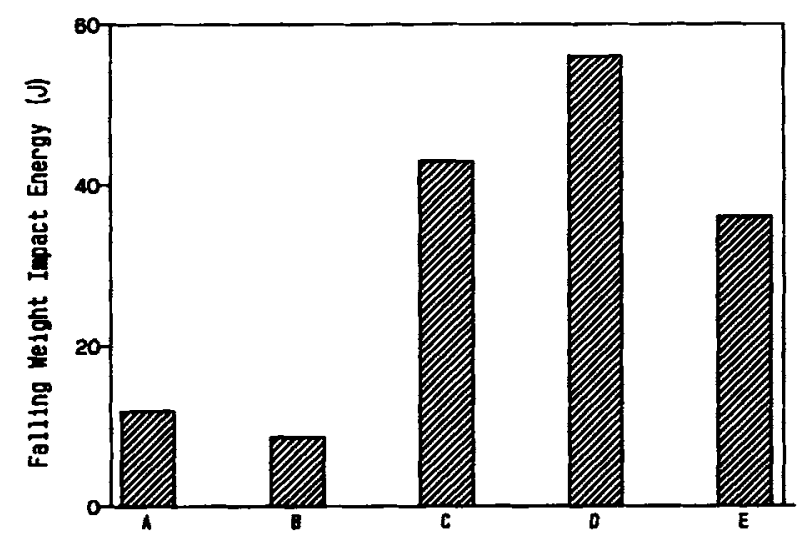

Figure 9 Total impact energy of the instrumental falling weight impact tests of various Phen / ABS $75 / 25$ blends: (A) Phen $/$ ABS $=75 / 25,11.95 \mathrm{~J}$; (B) Phen $/$ ABS $/$ SAG $=75 / 25 / 5,8.58 \mathrm{~J}$; (C) Phen $/$ ABS $/$ SAG $/$ Cat $=75 / 25 /$ $5 / 0.02,42.88 \mathrm{~J}$; (D) Phen $/$ ABS $/$ SAG $/$ Cat $=75 / 25 / 5 /$ $0.05,56.21 \mathrm{~J}$; (E) Phen $/$ ABS $/$ SAG $/$ Cat $=75 / 25 / 5 / 0.10$, $35.89 \jmath$. 


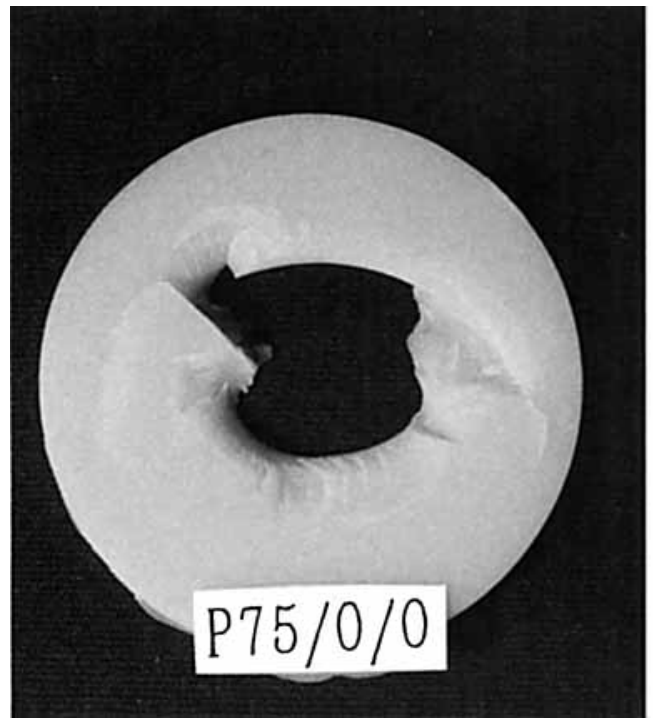

A

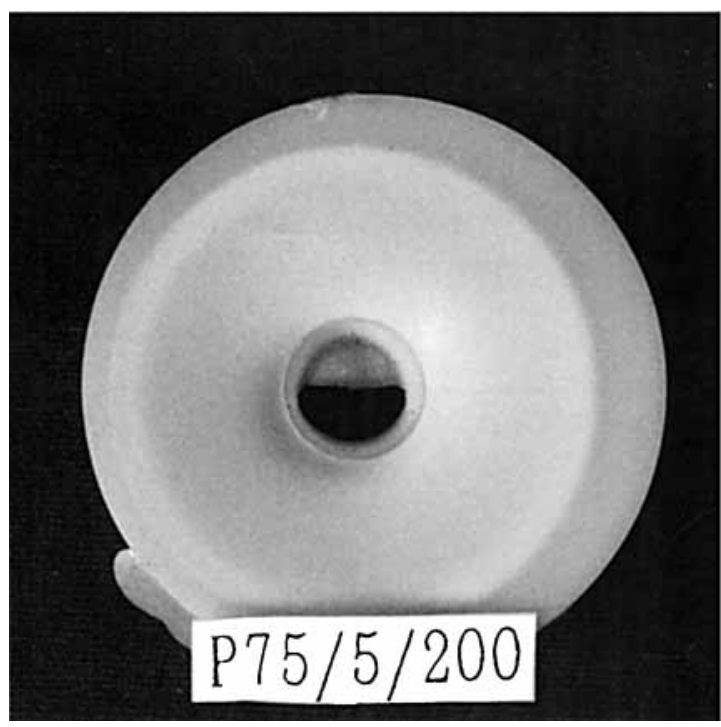

C

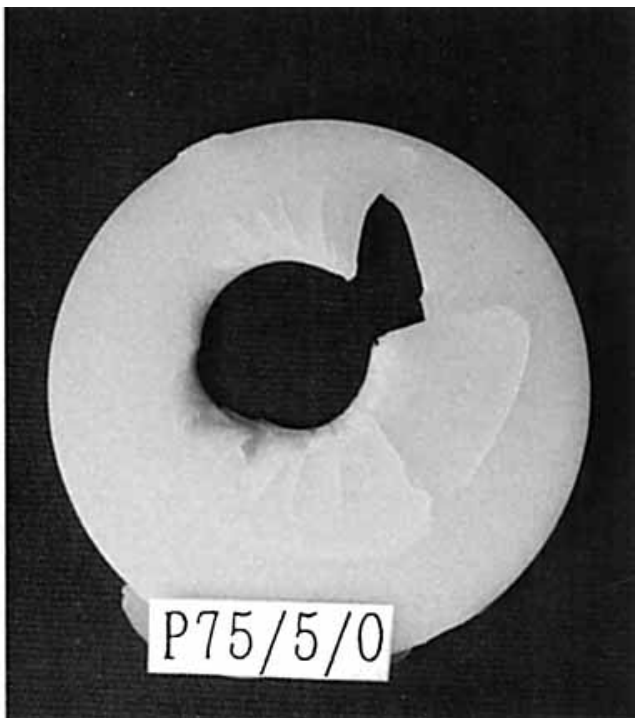

B

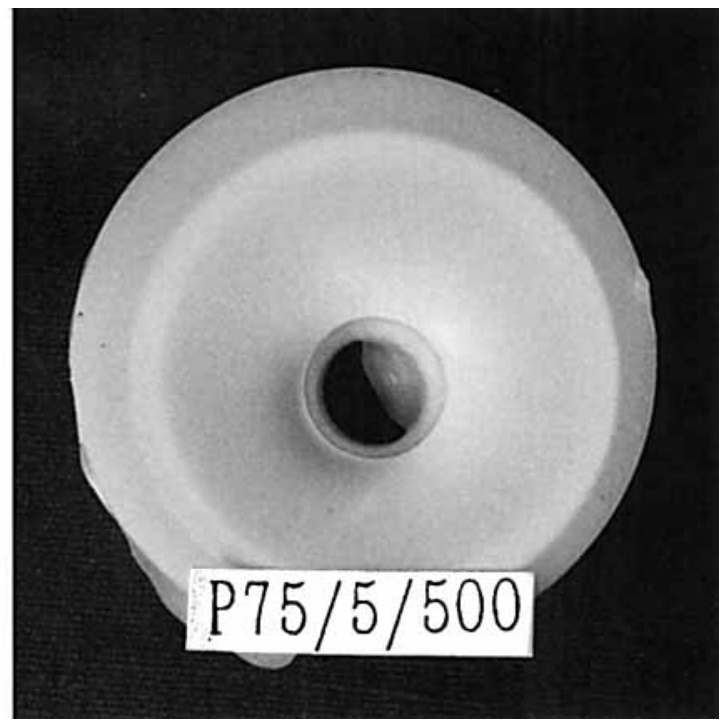

D

Figure 10 Photographs of the instrumental falling weight impact specimen: (A) Phen/ $\mathrm{ABS}=75 / 25$; (B) Phen $/ \mathrm{ABS} / \mathrm{SAG}=75 / 25 / 5$; (C) Phen $/$ ABS $/$ SAG $/$ Cat $=75 / 25 / 5 /$ 0.02 ; (D) Phen $/$ ABS $/$ SAG $/$ Cat $=75 / 25 / 5 / 0.05$.

for an applied load $P$ resulting in deflection $x$, we have,

$$
x / P=C(a),
$$

$a$ is the crack length. The energy absorbed will be the area under the triangular load-displacement diagram,

$$
W=0.5 P \cdot x=0.5 P^{2} \cdot C .
$$

For a specimen of uniform thickness $B$, then the 


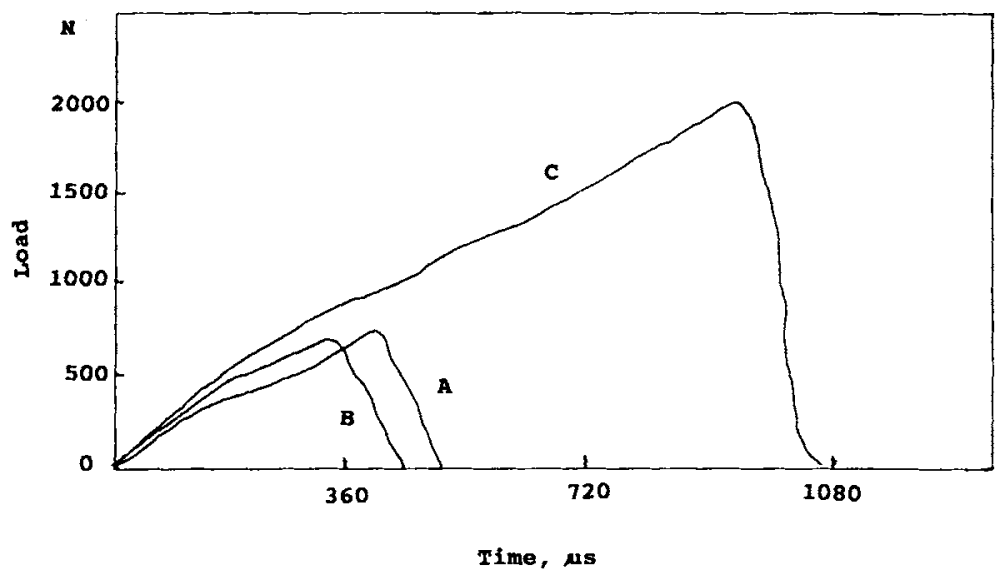

Figure 11 Instrumental falling weight impact load-time curves of various Phen / ABS 50/50 blends: (A) Phen $/ \mathrm{ABS}=50 / 50,4.65 \mathrm{~J}$; (B) Phen $/ \mathrm{ABS} / \mathrm{SAG}=50 / 50 / 5,3.95 \mathrm{~J}$; (C) Phen $/ \mathrm{ABS} / \mathrm{SAG} / \mathrm{Cat}=50 / 50 / 5 / 0.05,24.24 \mathrm{~J}$.

strain energy release rate $G$ is given by,

$$
G=(1 / B)(d W / d a)
$$

When $G=G_{\mathrm{c}}$, a critical value, the elastic and brittle fracture occurs.

$$
G_{\mathrm{c}}=\left(P^{2} / 2 B\right)(d C / d a) .
$$

From eqs. (2) and (4), we obtain the following relation,

$$
W=G_{\mathrm{c}} \cdot B \cdot D \cdot \phi
$$

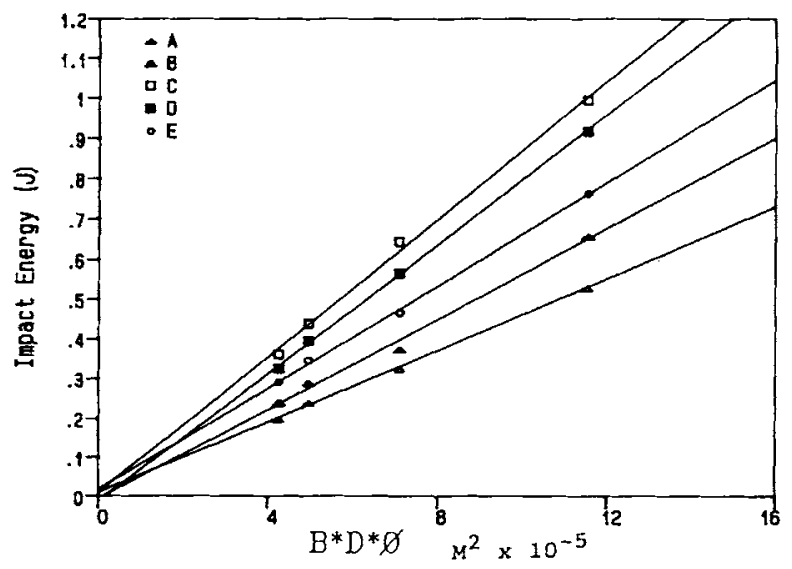

Figure 12 Plots of impact energy vs. $B \cdot D \cdot \phi$ of various Phen/ABS blends: (A) Phen /ABS $=75 / 25$; (B) Phen / ABS $/$ SAG $=75 / 25 / 5$; (C) Phen $/$ ABS $/$ SAG $/$ Cat $=75 /$ $25 / 5 / 0.05$; (D) Phen $/$ ABS $/$ SAG $/$ Cat $=75 / 25 / 2 / 0.05$; (E) Phen $/$ ABS $/ \mathrm{SAG} / \mathrm{Cat}=75 / 25 / 10 / 0.05$. and

$$
\phi=C / d C /[a / D]
$$

If $\phi$ is determined as a function of $[a / D]$ and the energy measured is plotted as function of $B \cdot D \cdot \phi$ for different geometries, a straight line of slope $G_{c}$ should result. The parameter $\phi$ can be determined experimentally by using eq. (6) or through a theoretical calculation. We employed the $\phi$ values previously reported ${ }^{34}$ in this study. Figure 12 shows the plots of impact energy versus $B \cdot D \cdot \phi$ for the same

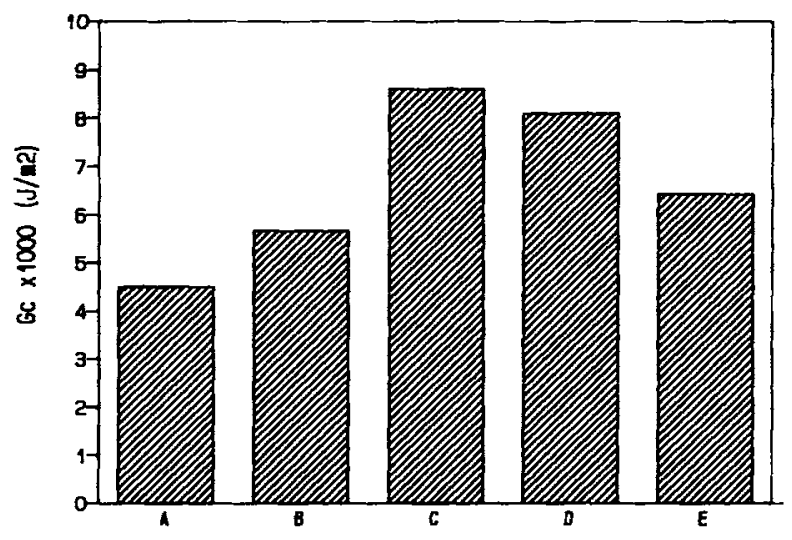

Figure 13 Critical strain energy release rate, $G_{c}$, of various Phen/ABS blends: (A) Phen $/ \mathrm{ABS}=75 / 25, G_{\mathrm{c}}$ $=4500 \mathrm{~J} / \mathrm{M}^{2}$; (B) Phen $/ \mathrm{ABS} / \mathrm{SAG}=75 / 25 / 5, G_{c}$ $=5660 \mathrm{~J} / \mathrm{M}^{2}$; (C) Phen/ABS $/ \mathrm{SAG} /$ Cat $=75 / 25 / 5 /$ $0.05, G_{c}=8600 \mathrm{~J} / \mathrm{M}^{2}$; (D) Phen $/ \mathrm{ABS} / \mathrm{SAG} / \mathrm{Cat}=75 /$ $25 / 2 / 0.05, G_{\mathrm{c}}=8090 \mathrm{~J} / \mathrm{M}^{2}$; (E) Phen $/ \mathrm{ABS} / \mathrm{SAG} / \mathrm{Cat}$ $=75 / 25 / 10 / 0.05, G_{c}=6430 \mathrm{~J} / \mathrm{M}^{2}$. 
set of blends as the Izod impact tests. The $G_{\mathrm{c}}$ values obtained from the slopes in Figure 12 are shown in Figure 13. The order of $G_{\mathrm{c}}$ shown in Figure 13 is identical to previous lzod impact strength or tensile properties. Again, the optimized catalyst content to achieve maximum $G_{\mathrm{c}}$ is at $0.05 \mathrm{phr}$.

\section{CONCLUSIONS}

Phenoxy and ABS are an immiscible and incompatible polymer pair in terms of microstructure and mechanical properties. Addition of the in situ compatibilizer, SAG copolymer, does not shows substantial improvement over the noncompatibilized one. Without the presence of a proper catalyst, the reaction between epoxide from SAG and aliphatic$\mathrm{OH}$ from phenoxy is very slow and the amount of the expected copolymer produced is insignificant. Sodium lauryl sulfonate is a suitable catalyst to promote reaction between phenoxy and SAG under melt conditions. This in situ formed copolymer acts as compatibilizer for the polymer blends of phenoxy and ABS.

This research project was financially supported by the National Science Council of the Republic of China under contract number NSC 81-0405-E-009-04.

\section{REFERENCES}

1. I. Mondragon, M. Gaztelumendi, and J. Nazabal, Polym. Eng. Sci., 26, 1478 (1986).

2. I. Mongragon, M. Gaztelumendi, and J. Nazabal, Polym. Eng. Sci., 28, 1126 (1988).

3. X. Yang, P. C. Painter, and M. M. Coleman, Macromolecules, 25, 4996 (1992).

4. W. H. Christiansen, D. R. Paul, and J. W. Barlow, J. Appl. Polym. Sci., 34, 537 (1987).

5. J. H. Chu and F. C. Chang, Proc. of the Annual Conf. of the Chinese Soc. for Mater. Sci., Taiwan, p. 1021, 1990.

6. M. M. Coleman, X. Yang, P. C. Painter, and J. F. Graf, Macromolecules, 25, 4414 (1992).

7. J. I. Eguiazabal, J. J. Iruin, M. Cortazar, and G. M. Guzman, J. Appl. Polym. Sci., 32, 5945 (1986).

8. J. E. Harris, S. H. Goh, D. R. Paul, and J. W. Barlow, J. Appl. Polym. Sci., 27, 839 (1982).
9. L. M. Robeson and A. B. Furtek, J. Appl. Polym. Sci., 23, 645 (1979).

10. J. I. Eguiazabal and J. Nazabal, J. Mater. Sci., 25, 1522 (1990).

11. M. M. Coleman and E. J. Moskala, Polymer, 24, 251 (1983).

12. L. M. Robeson, W. F. Hale, and C. N. Merriam, Macromolecules, 14, 1644 (1981).

13. J. H. Chu and F. C. Chang, Proc. of the 13th ROC Polym. Symp., Taiwan, p. 326, 1990.

14. Y. S. Soh, J. Appl. Polym. Sci., 45, 1831 (1992).

15. J. S. Chiou, D. R. Paul, J. Appl. Polym. Sci., 42, 279 (1991).

16. R. Erro, M. Gaztelumendi, and J. Nazabal, J. Appl. Polym. Sci., 45, 339 (1992).

17. Q. Guo, J. Huang, B. Li, T. Chen, H. Zhang, and Z. Feng, Polymer, 32, 58 (1991).

18. M. Kodama, Polym. Eng. Sci., 32, 267 (1992).

19. E. J. Moskala and M. M. Coleman, Polym. Commun., 24, 206 (1983).

20. M. A. Iriarte, J. J. Iruin, and J. I. Eguiazabal, J. Mater. Sci., 24, 1021 (1989).

21. C. G. Seefried, Jr., J. V. Koleske, and F. C. Critchfield, Polym. Eng. Sci., 16, 771 (1976).

22. Q. Guo, J. Huang, and T. Chen, Polym. Bull., 20, 517 (1988).

23. M. Xanthos, Polym. Eng. Sci., 28, 1392 (1988).

24. L. A. Utracki, Polymer Alloys and Blends, Hanser Publishers, Munich, 1990, p. 124.

25. B. S. Chang, F. C. Chang, N. C. Chu, and C. S. Huang, Proc. of the 15th ROC Polym. Symp., Taiwan, p. 425, 1992.

26. C. T. Maa and F. C. Chang, J. Appl. Polym. Sci., 49, 913 (1993).

27. W. B. Liu and F. C. Chang, Polymer Preprints, 34 (2), 803 (1993)

28. F. C. Chang and Y. C. Hwu, Polym. Eng. Sci., 31, 1509 ( 1991 ).

29. H. H. Chang, J. S. Wu, and F. C. Chang, Proc. of the 4th Asian Chem. Cong., Beijing, p. 699 (1991).

30. F. C. Chang and M. Y. Yang, Polym. Eng. Sci., 30, $543(1990)$.

31. F. C. Chang, J. S. Wu, and L. H. Chu, J. Appl. Polym. Sci., 44, 491 (1992).

32. J. S. Wu, S. C. Shen, and F. C. Chang, J. Appl. Polym. Sci., to appear.

33. H. R. Brown, J. Mater. Sci., 8, 941 (1973).

34. G. P. Marshall, J. G. Williams, and C. E. Turner, J. Mater. Sci., 8, 949 (1973).

Received June 10, 1993

Accepted July 22, 1993 